\title{
Th17/Treg-cell balance in the peripheral blood of pregnant females with a history of recurrent spontaneous abortion receiving progesterone or cyclosporine $A$
}

\author{
SONGCUN WANG ${ }^{1,2 *}$, MENGDIE LI ${ }^{1,2^{*}}$, FENGRUN SUN $^{1,2}$, CHUNQIN CHEN $^{1,2}$, \\ JIANGFENG YE ${ }^{2}$, DAJIN LI ${ }^{1,2}$, JINFENG QIAN ${ }^{1,2}$ and MEIRONG DU ${ }^{1,2}$ \\ ${ }^{1}$ NHC Key Laboratory of Reproduction Regulation (Shanghai Institute of Planned Parenthood Research); \\ ${ }^{2}$ Shanghai Key Laboratory of Female Reproductive Endocrine Related Diseases, Hospital of Obstetrics and Gynecology, \\ Fudan University Shanghai Medical College, Shanghai 200080, P.R. China
}

Received January 29, 2019; Accepted June 27, 2019

DOI: $10.3892 /$ etm.2020.9469

\begin{abstract}
A successful pregnancy requires the maternal immune system to accept a fetus expressing allogeneic paternal antigens and provide competent responses to infections. Accordingly, maternal-fetal immune abnormalities may have an important role in the development of recurrent spontaneous abortion (RSA). Ever since the establishment of the association between immunologic abnormalities and RSA, various types of immune therapy to restore normal immune homeostasis have been increasingly developed. Although previous studies have focused on the maternal-fetal interface, non-invasive examination is of great importance in clinical practice. The present study investigated the balance between type-17 T-helper (Th17) and T-regulatory (Treg) cells in the peripheral blood to improve the current understanding of the pathogenesis of RSA. Imbalances in Th17/Treg cells and associated molecular profiles were observed in patients with RSA. Furthermore, it was determined that the immunosuppressant cyclosporine A reduced the proportion of Th17 cells and promoted Treg-cell dominance by upregulating the expression of co-inhibitory molecules in pregnant females with a history of RSA. Progesterone, the traditional maternal-care drug, also had a certain immunomodulatory role through restoring the levels of several co-inhibitory molecules (including T-cell immunoglobulin mucin family member-3, programmed cell
\end{abstract}

Correspondence to: Dr Meirong Du or Dr Jinfeng Qian, NHC Key Laboratory of Reproduction Regulation (Shanghai Institute of Planned Parenthood Research), Fudan University Shanghai Medical College, 1326 Pingliang Road, Shanghai 200080, P.R. China

E-mail: dmrlq1973@sina.cn

E-mail: qjf2003@163.com

*Contributed equally

Key words: recurrent spontaneous abortion, cyclosporine A, progesterone, Th17 cells, Treg cells death-1 and cytotoxic T-lymphocyte associated protein-4) in the treatment of RSA. Changes in these immune molecules within the maternal peripheral blood may be indicators for monitoring pregnancy and prediction of RSA.

\section{Introduction}

Recurrent spontaneous abortion (RSA), defined as two or more consecutive pregnancy losses prior to 20 weeks of gestation, may seriously compromise not only the physical but also psychological well-being of women. Although parental or embryonic karyotype anomalies, infection-associated factors, endocrine disorders, uterine anatomic abnormalities and anti-phospholipid syndrome have been acknowledged as the common causes of RSA, approximately half of the cases still remain unexplained (1). The development of the allogeneic fetus in the maternal uterus represents an immunological paradox. A successful pregnancy requires the maternal immune system to accept the fetus expressing allogeneic paternal antigens and provide competent responses to infections. Thus, maternal-fetal immune abnormalities might have an important role in the development of RSA (2).

The modulation of the maternal $\mathrm{CD} 4^{+} \mathrm{T}$-cell response to fetal antigens has been considered an important component of maternal-fetal tolerance during pregnancy. Upon encountering antigens on the surface of antigen-presenting cells or being driven by a set of cytokines, naive $\mathrm{CD} 4^{+} \mathrm{T}$ cells are able to differentiate into distinct subsets, including type-1 T-helper (Th1), Th2, Th17 and T-regulatory (Treg) cells (3). While a pro-inflammatory microenvironment is required for trophoblastic growth and invasion (4), Th1 cytokines, including tumor necrosis factor- $\alpha$ and interferon- $\gamma$, have deleterious effects on pregnancy (5). For numerous years, it has been thought that a shift in the maternal immune response toward a Th2 bias is crucial for maintaining a successful pregnancy (6).

Th17 cells have a critical role in inducing inflammation, while abnormal Th17-cell levels have been associated with the pathogenesis of autoimmune diseases (7). Treg cells express anti-inflammatory cytokines, including interleukin (IL)-10 and transforming growth factor (TGF)- $\beta 1$, which dampen an 
excessively effective immune response (8). Recent studies have indicated that the balance between Treg and Th17 cells is also important for maintaining a normal pregnancy, while a shift in the Th17/Treg ratio toward Th17 cells has been suggested to cause several pregnancy-associated diseases, including RSA, pre-eclampsia and gestational diabetes mellitus (9-11).

In addition to the signal from antigen recognition, T-cell activation requires a second signal from co-stimulatory molecules, which maybe either positive or negative $(12,13)$. Among the inhibitory co-stimulatory molecules, cytotoxic T-lymphocyte-associated protein 4 (CTLA-4), T-cell immunoglobulin mucin-3 (Tim-3) and programmed cell death-1 (PD-1) have attracted much attention regarding their roles in regulating Th17- and Treg-cell function to maintain a balanced immune response (14-17). For instance, Tim-3 has been associated with a shift in the balance from Th17 and Treg cells to Treg dominance (16). The PD-1 signaling pathway has been demonstrated to have an important role in Th17-cell function during pregnancy (18). Furthermore, PD-1 and CTLA-4 are critical for the suppressive activity of Treg cells $(19,20)$, while PD- $1^{+}$Tim- $3^{+}$Treg cells exhibit an increased effector function (21). Previous studies by our group revealed that the expression of these three molecules was lower in human miscarriages than in normal pregnancies, while their blockade during the implantation window increased maternal rejection of the fetus in CBA/Jx BALB/c matings $(22,23)$.

Progesterone (P4), the most common medication for fetal protection in RSA, is a multifunctional female hormone that promotes breast development, ovulation and pregnancy maintenance; it is mainly secreted by ovarian granulosa cells and the corpus luteum during non-pregnancy and by the placenta during pregnancy (24). While the mechanisms remain to be fully elucidated, $\mathrm{P} 4$ has been reported to dampen immune responses to fetal and maternal antigens (25). As an immunosuppressant, cyclosporine A (CsA) has been widely used in organ transplantation (26). CsA not only induces Th2 immuno-dominance at the maternal-fetal interface, but also promotes trophoblastic proliferation and invasion, further promoting placental formation (27). This immunosuppressive agent, categorized as pregnancy category $\mathrm{C}$ by the Food and Drug Administration, has been utilized for the treatment of RSA at our hospital (Hospital of Obstetrics and Gynecology, Fudan University Shanghai Medical College) since 2005.

To the best of our knowledge, studies on the effects of P4 or CsA on the proportion of Th17 and Treg cells within the peripheral blood of patients with RSA are rare. Therefore, the present study aimed to investigate the effects of P4 or CsA therapy on the Th17/Treg cell balance and the expression of associated molecules in the peripheral blood of pregnant females with a history of RSA.

\section{Materials and methods}

Study population. For the present study, subjects aged between 20 and 39 years were recruited at the Obstetrics and Gynecology Hospital of Fudan University (Shanghai, China) between July 2018 and December 2018. The clinical characteristics of the enrolled subjects are summarized in Table I. The cohort included 30 females with normal early pregnancy (NP group),
25 with RSA (RSA group), 27 pregnant females with a history of RSA receiving progesterone ( $\mathrm{P} 4$ group) and 24 pregnant females with a history of RSA receiving cyclosporine A (CsA group). Subjects with RSA included those undergoing spontaneous abortion and who also had a history of two or more consecutive spontaneous abortions before 20 weeks (the mean pregnancy week was $6.28 \pm 0.09$ ) of gestation without known causes (including parental or embryonic karyotype anomalies, uterine anatomic abnormalities, infection-associated factors, endocrine disorders and antiphospholipid syndrome). The subjects in the NP group (the mean pregnancy week was $6.26 \pm 0.11$ ) had at least one successful pregnancy and no history of spontaneous abortions. None of the subjects had any history of autoimmune diseases or immunotherapy, renal or liver diseases, alcohol addiction, smoking or vaccination within 3 months prior to consultation. Approval of the present study was obtained from the ethics committee of the Obstetrics and Gynecology Hospital of Fudan University (Shanghai, China) and all subjects provided written informed consent.

In this open-label (i.e. non-blind) study, pregnant females with a history of RSA were randomly allocated to the P4 or CsA group after a positive human chorionic gonadotropin urine test. The P4 group received dydrogesterone (Abbott) $10 \mathrm{mg}$ Q8 h orally, while the CsA group received CsA (Sanofi) $50 \mathrm{mg}$ Q8 h orally. After 10 days, venous blood was collected (at this time the mean pregnancy week of P4 group was $6.16 \pm 0.1$, the

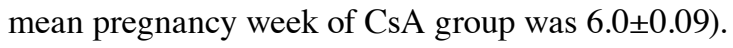

Separation of peripheral blood mononuclear cells. Peripheral blood mononuclear cells were isolated from $5 \mathrm{ml}$ heparinized venous blood using Ficoll-Hypaque density gradient centrifugation (573 x g at room temperature for $20 \mathrm{~min}$ ). Cells were collected at the interface and washed with PBS. Cells were cultured in RPMI 1640 (HyClone; GE Healthcare Life Sciences) supplemented with $10 \%$ fetal bovine serum (HyClone; GE Healthcare Life Sciences), $100 \mathrm{U} / \mathrm{ml}$ penicillin, $100 \mu \mathrm{g} / \mathrm{ml}$ streptomycin and $1 \mu \mathrm{g} / \mathrm{ml}$ amphotericin B. Phorbol 12-myristate 13-acetate (PMA; 50 ng/ml; BioLegend, Inc.), ionomycin (1 $\mu \mathrm{g} / \mathrm{ml}$; BioLegend, Inc.) and brefeldin A (10 mg/ml; BioLegend, Inc.) were added to the culture for $4 \mathrm{~h}$ prior to intracellular cytokine analysis.

Flow cytometric analysis. Cell surface molecular expression and intracellular cytokine production were evaluated using flow cytometry (FCM). Alexa eFluor ${ }^{\circledR}$ 488-conjugated anti-human forkhead box p3 (Foxp3; cat. no. 320112), phycoerythrin (PE)-conjugated anti-human CD366 Tim-3 (cat. no. 345006) or CD152 CTLA-4 (cat. no. 349906), $\mathrm{PE} /$ cyanine 7-conjugated anti-human IL-17A (cat. no. 512315), allophycocyanine-conjugated anti-human CD279 PD-1 (cat. no. 329908) or LAP TGF- $\beta 1$ (cat. no. 349608), Brilliant Violet 421-conjugated anti-human IL-10 (cat. no. 501422) and Brilliant Violet 510-conjugated anti-human CD4 (cat. no. 317444) antibodies (BioLegend, Inc.) were used. For intracellular staining, cells were fixed and permeabilized using the Fix/Perm kit (cat. no. 421401/2). The fix/perm kit and all antibodies aforementioned were purchased from BioLegend, Inc. Flow cytometry was performed on a Beckman-Coulter CyAn ADP cytometer and the results were analyzed using FlowJo software (version 7.6; FlowJo, LLC). 
Table I. Clinical characteristics of enrolled subjects.

\begin{tabular}{|c|c|c|c|c|c|}
\hline Subjects $^{\mathrm{b}}$ & $\mathrm{NP}(\mathrm{n}=30)$ & $\operatorname{RSA}(\mathrm{n}=25)$ & $\mathrm{P} 4(\mathrm{n}=27)$ & $\operatorname{CsA}(n=24)$ & P-value \\
\hline Age mean (years) ${ }^{\mathrm{a}}$ & $28.07 \pm 0.86$ & $28.96 \pm 0.98$ & $29.26 \pm 0.83$ & $29 \pm 1.0$ & 0.80 \\
\hline Age range (years) & $20-39$ & $22-38$ & $23-38$ & $21-39$ & N/A \\
\hline Previous spontaneous abortions ${ }^{\mathrm{a}}$ & - & $2.92 \pm 0.22$ & $2.85 \pm 0.15$ & $2.95 \pm 0.23$ & 0.93 \\
\hline Pregnancy week (HCG urine test) ${ }^{\mathrm{a}}$ & - & - & $4.72 \pm 0.09$ & $4.58 \pm 0.09$ & 0.33 \\
\hline Pregnancy week (venous blood was collected) ${ }^{\mathrm{a}}$ & $6.26 \pm 0.11$ & $6.28 \pm 0.09$ & $6.16 \pm 0.1$ & $6.0 \pm 0.09$ & 0.31 \\
\hline
\end{tabular}

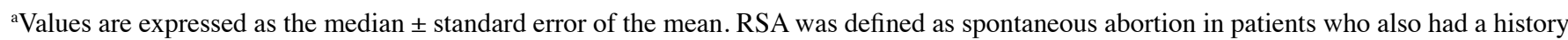

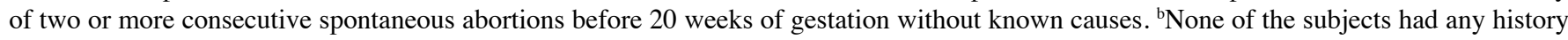
of treatment. Groups: NP, normal early pregnancy; RSA; patients with RSA; P4, pregnant females with a history of RSA treated with P4; CsA, pregnant females with a history of RSA treated with CsA. N/A, not applicable; CsA, cyclosporin A; RSA, recurrent spontaneous abortion; P4, progesterone; HCG, human chorionic gonadotropin.
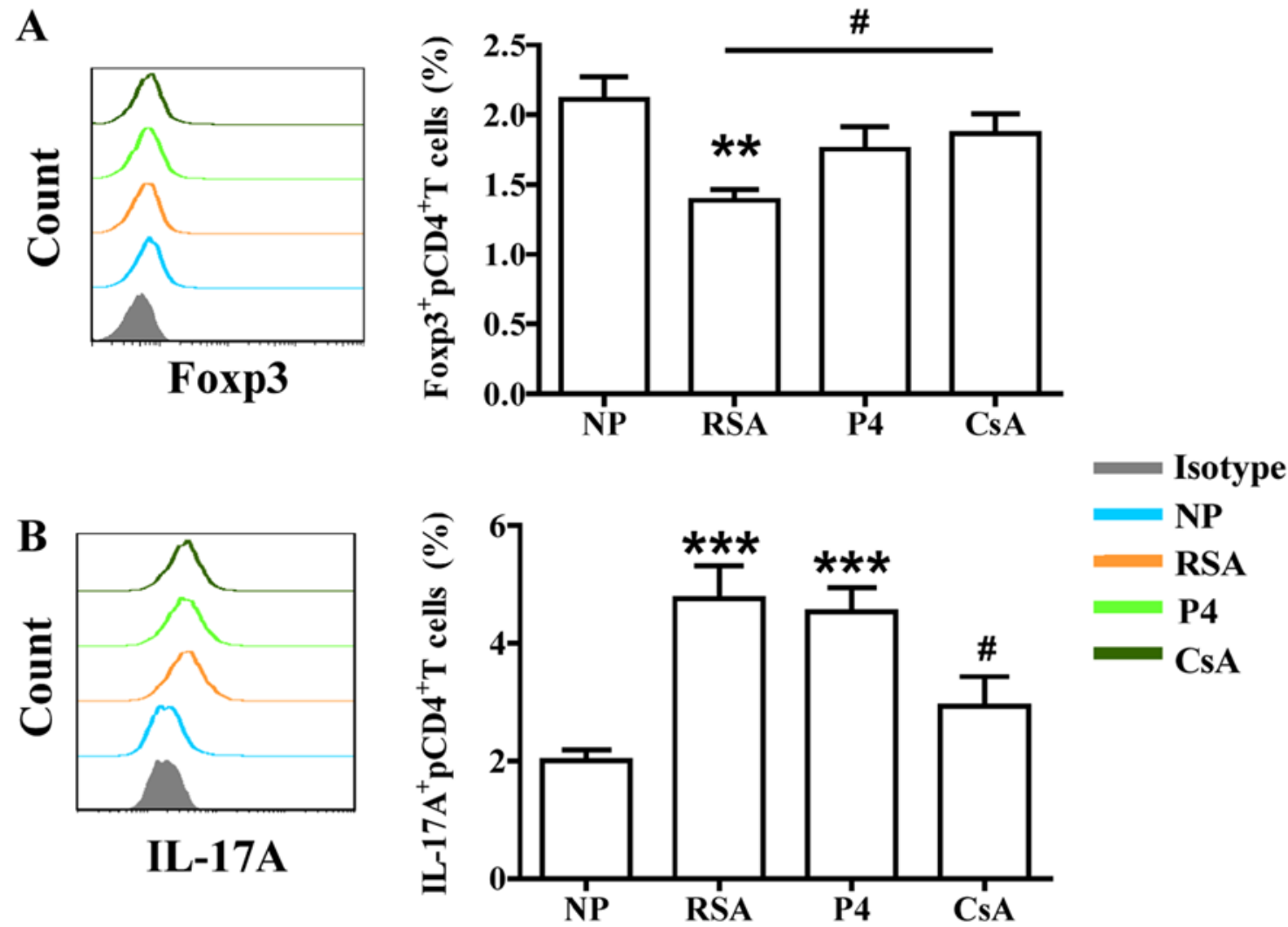

Figure 1. Effects of P4 and CsA on the percentage of Treg and Th17 cells in peripheral blood. (A) Compared with that in the NP group, the subjects in the RSA group had a significantly lower percentage of Treg cells in the peripheral blood. CsA significantly increased the percentage of Treg cells. (B) Compared with that in the NP group, the subjects in the RSA group had a higher percentage of Th17 cells in the peripheral blood. CsA decreased the percentage of Th17 cells. Groups: NP, normal early pregnancy; RSA; patients with RSA; P4, pregnant females with a history of RSA treated with P4; CsA, pregnant females with a history of RSA treated with CsA. ${ }^{* *} \mathrm{P}<0.01,{ }^{* * *} \mathrm{P}<0.001 \mathrm{vs.} \mathrm{NP}$; ${ }^{*} \mathrm{P}<0.05$ vs. RSA. CsA, cyclosporine A; Treg cells, T-regulatory cells; Th17 cells, type-17 T-helper cells; RSA, recurrent spontaneous abortion; Fox, forkhead box; IL, interleukin; P4, progesterone.

Statistical analysis. All variables were normally distributed in this study. Thus, variables were presented as means and standard deviation (SD). One-way ANOVA was used to evaluate differences. $\mathrm{P}<0.05$ was considered to indicate a statistically significant difference. In cases where $\mathrm{P}<0.05$ after ANOVA, Bonferroni's post hoc test was performed to determine differences between each group. All analyses were carried out using the GraphPad Prism 7 software (GraphPad Software, Inc.).

\section{Results}

CsA significantly increases the percentage of Treg cells and decreases the percentage of Th17 cells in the peripheral blood of pregnant females with a history of RSA. As presented in Fig. 1, the patients from the RSA group had a significantly decreased percentage of Treg cells $\left(\mathrm{CD} 4^{+} \mathrm{Foxp} 3^{+}\right.$cells) and an increased percentage of Th17 cells (CD4+IL-17A ${ }^{+}$cells) in the peripheral blood compared with those in the NP group. 
A
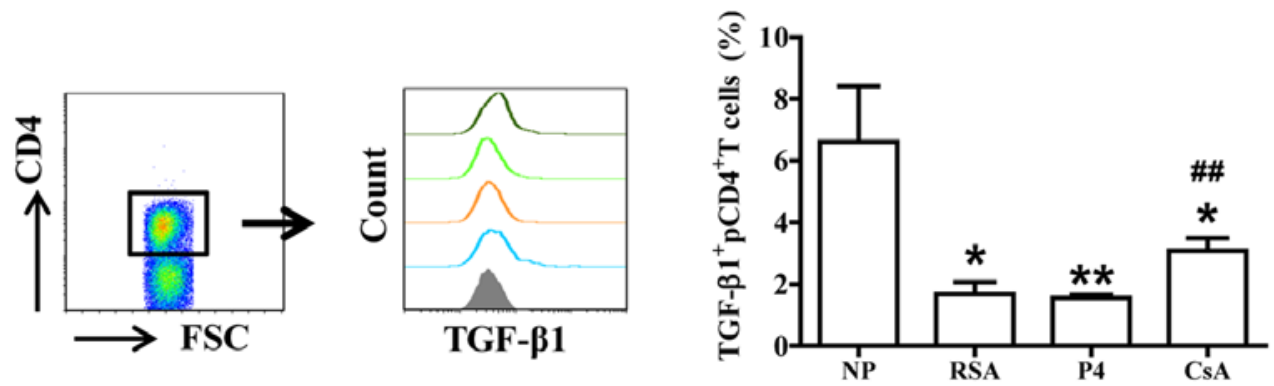

B
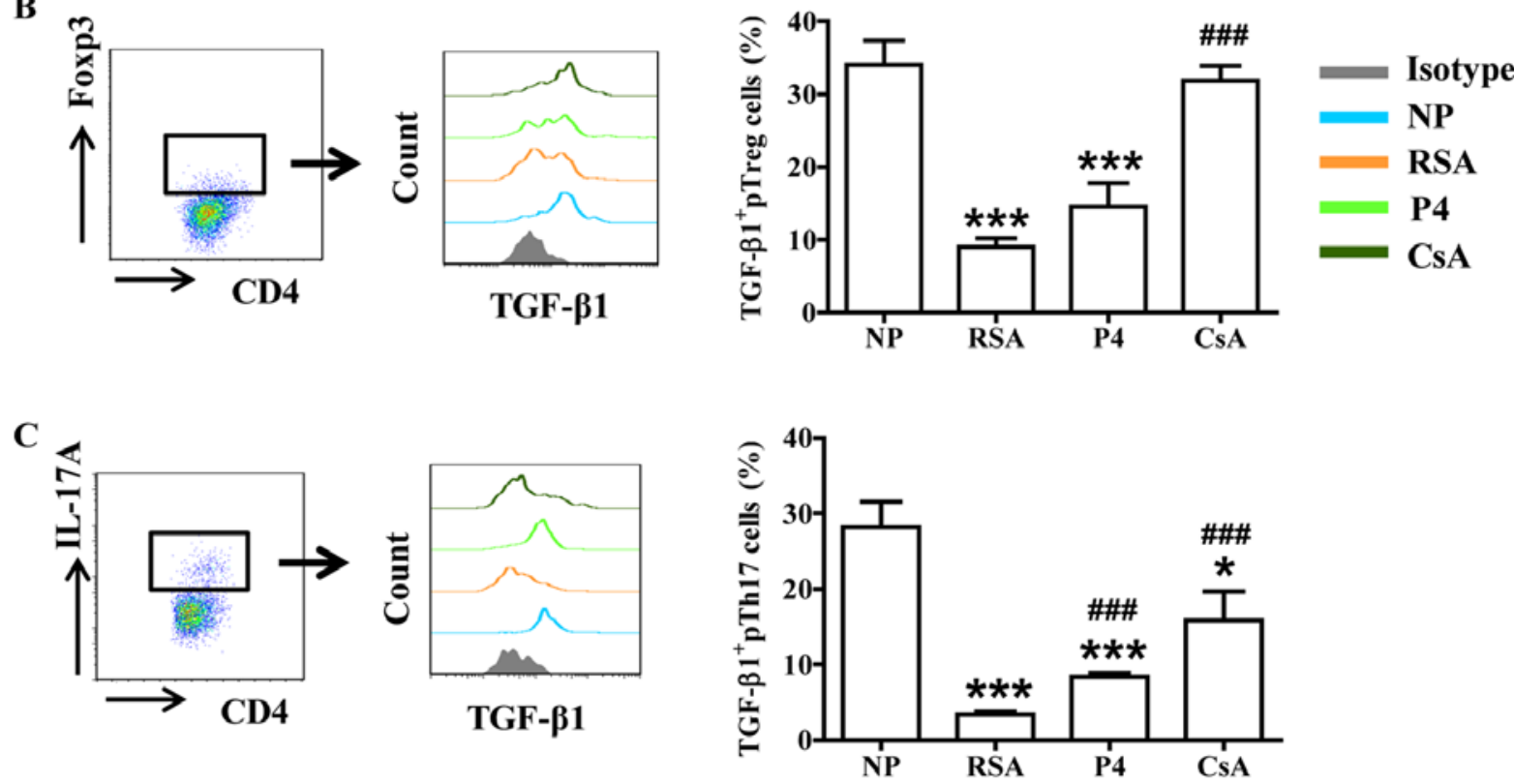

Figure 2. CsA promotes TGF- $\beta 1$ expression in peripheral blood cells of pregnant females with RSA. Compared with that in the NP group, the production of TGF- $\beta 1$ in (A) CD4 ${ }^{+}$T cells, (B) Treg cells and (C) Th17 cells in the peripheral blood of subjects in the RSA group was decreased. While CsA and P4 promoted the expression of TGF- $\beta 1$ in Th17 cells, P4 had no effect on TGF- $\beta 1$ production by CD4 ${ }^{+}$T cells and Treg cells. Groups: NP, normal early pregnancy; RSA; patients with RSA; P4, pregnant females with a history of RSA treated with $\mathrm{P} 4$; CsA, pregnant females with a history of RSA treated with CsA. ${ }^{*}<0.05$, ${ }^{* *} \mathrm{P}<0.01,{ }^{* * *} \mathrm{P}<0.001$ vs. NP; ${ }^{\#} \mathrm{P}<0.01,{ }^{\# \# \#} \mathrm{P}<0.001$ vs. RSA. CsA, cyclosporine A; pTreg cells, peripheral T-regulatory cells; Th17 cells, type-17 T-helper cells; RSA, recurrent spontaneous abortion; P4, progesterone; Fox, forkhead box; IL, interleukin; TGF, transforming growth factor; FSC, forward scatter.

Of note, CsA increased the percentage of Treg cells, while decreasing the percentage of Th17 cells in the peripheral blood of pregnant females with a history of RSA compared with the percentages in the RSA group. However, P4 exhibited no significant effect on the proportion of Th17 or Treg cells in pregnant females with a history of RSA compared with the proportions in the RSA group.

CsA promotes TGF- $\beta 1$ and IL-10 production in peripheral blood cells of pregnant females with a history of RSA. To assess whether CsA or $\mathrm{P} 4$ regulates the biological functions of Th17/Treg cells in pregnant females, the production of cytokines (associated with Treg- and Th17-cell function) by peripheral $\mathrm{T}$ cells was first analyzed. Compared with those in the NP group, patients in the RSA group exhibited significantly lower TGF- $\beta 1$ and IL-10 expression in total peripheral (p) $\mathrm{CD}^{+} \mathrm{T}$ cells, pTreg cells and pTh17 cells. However, CsA increased TGF- $\beta 1$ and IL-10 expression in the peripheral blood cells of pregnant females with a history of RSA, whereas $\mathrm{P} 4$ promoted only TGF- $\beta 1$ expression in pTh17 cells (Figs. 2 and 3).
Effects of CsA and P4 on CTLA-4, Tim-3 and PD-1 expression in peripheral blood cells of pregnant females with a history of $R S A$. In addition to the signal from the T-cell receptor complex after antigen recognition, T-cell activation requires a second signal from co-signaling molecules. Recently, the role of co-signaling molecules, particularly co-inhibitory molecules, in maternal-fetal immunity has been explored (28). The effects of CsA and P4 on CTLA-4, Tim-3 and PD-1 expression in the peripheral blood cells of pregnant females were then assessed. As presented in Figs. 4-6, the patients in the RSA group demonstrated significantly lower CTLA-4, Tim-3 and PD-1 expression in the peripheral blood cells, but the expression of Tim-3 and PD-1 remained unchanged on Th17 cells, compared with those in the NP group. CsA increased CTLA-4 expression in total pCD $4^{+} \mathrm{T}$ cells, pTreg cells and pTh17 cells, whereas P4 only promoted CTLA-4 expression in total pCD4 ${ }^{+} \mathrm{T}$ cells and pTh17 cells of pregnant females with a history of RSA (Fig. 4). CsA and $\mathrm{P} 4$ increased Tim- 3 expression on total $\mathrm{pCD}^{+} \mathrm{T}$ cells, pTreg cells and pTh17 cells (Fig. 5). Furthermore, P4 increased PD-1 expression in pTreg cells, while CsA had no effect on PD-1 expression in peripheral blood cells (Fig. 6). 
A

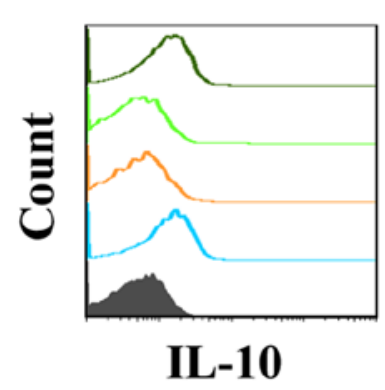

B

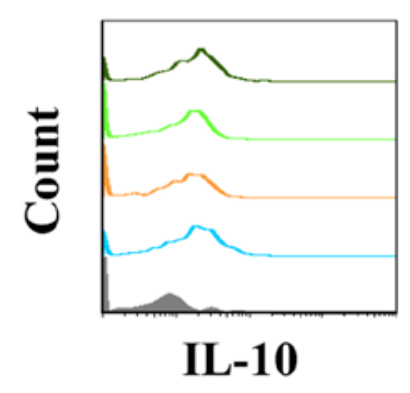

C

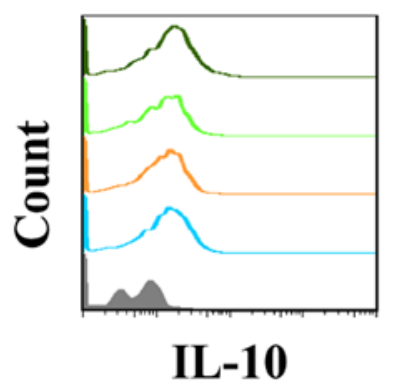

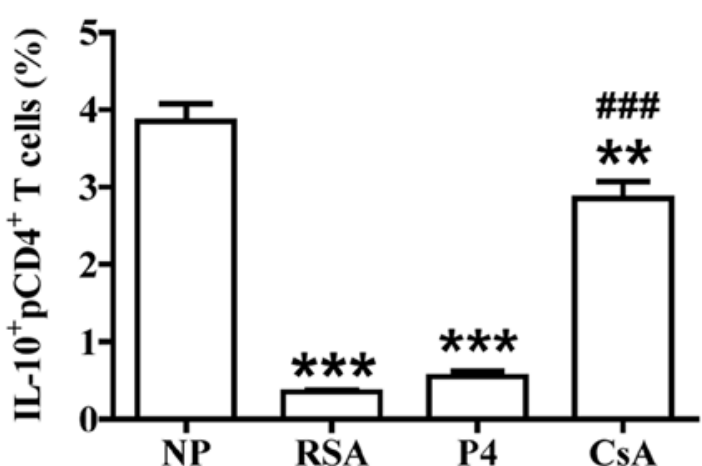
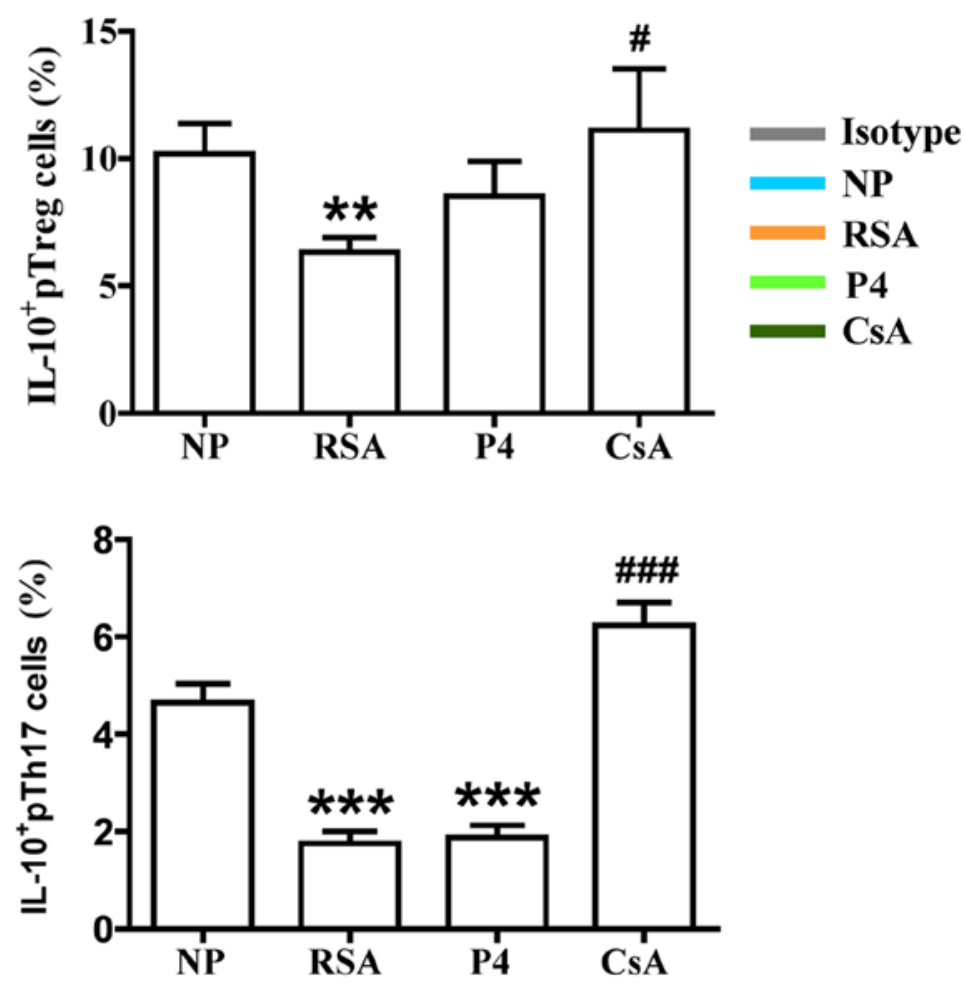

Figure 3. CsA promotes IL-10 expression in peripheral blood cells of pregnant females with RSA. Compared with that in the NP group, the production of IL-10 in (A) CD4 $4^{+}$T cells, (B) Treg cells and (C) Th17 cells in the peripheral blood of subjects in the RSA group was decreased. While CsA promoted the production of IL-10, P4 had no effect on it. Groups: NP, normal early pregnancy; RSA; patients with RSA; P4, pregnant females with a history of RSA treated with P4; CsA, pregnant females with a history of RSA treated with CsA. ${ }^{* *} \mathrm{P}<0.01,{ }^{* * *} \mathrm{P}<0.001 \mathrm{vs}$. NP; ${ }^{\#} \mathrm{P}<0.05,{ }^{\# \# \#} \mathrm{P}<0.001$ vs. RSA. CsA, cyclosporin A; pTreg cells, peripheral T-regulatory cells; Th17 cells, type-17 T-helper cells; RSA, recurrent spontaneous abortion; P4, progesterone; IL, interleukin.

\section{Discussion}

As a semi-allograft, the human fetus is antigenically foreign to the mother. Accordingly, a successful pregnancy relies on the fine maternal-fetal immune balance between immune tolerance, allowing allogeneic fetal trophoblasts to invade maternal tissues and immune defense against a variety of pathogens. Disruption of this immune balance is thought to be associated with several pregnancy-associated complications, including RSA, preeclampsia and fetal intrauterine growth restriction (2). The present study also confirmed that imbalance of Th17/Treg cells and associated molecule is linked to RSA.

$\mathrm{P} 4$, the traditional treatment for RSA, inhibits uterine contraction and improves luteal-phase support and utero-placental circulation (29). Chien et al (30) indicated that P4 may also regulate immunity and inhibit T-cell activation through a non-classical steroid receptor pathway during pregnancy. In early 1995, Piccinni et al (31) proposed that P4 was beneficial to $\mathrm{Th} 2$ differentiation of $\mathrm{CD} 4{ }^{+} \mathrm{T}$ cells. Furthermore, Mjosberg et al (32) revealed that P4 regulated Treg-cell functions in human pregnancy, while Lee et al (33) reported that P4 inhibited Th17-cell differentiation and reduced the expression of associated functional molecules, including IL-17A. However, the present study revealed no significant differences in the proportion of Treg and Th17 cells in the peripheral blood between the P4 and RSA groups. This may be due to differences in the gestational stages tested and the fact that certain studies were performed using established cell clones.

While certain studies have suggested the involvement of Th17 cells in pregnancy-associated pathologies (34), a study by our group (35) and Wu et al (36) suggested that Th17 cells serve important roles in regulating trophoblast 
A

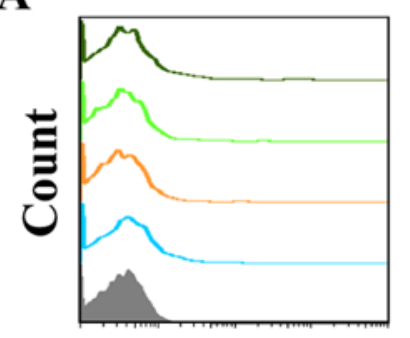

CTLA-4

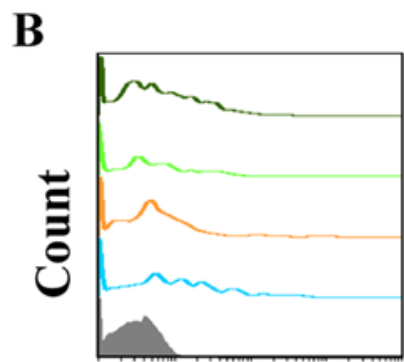

CTLA-4

C

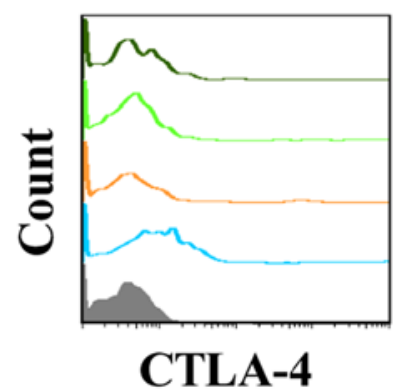

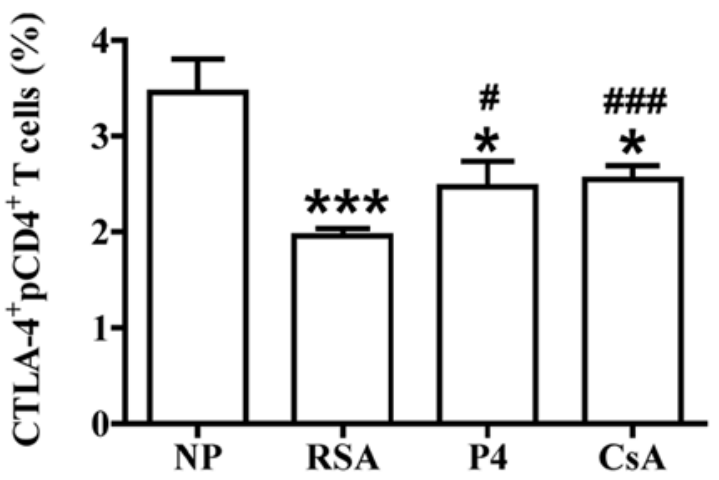
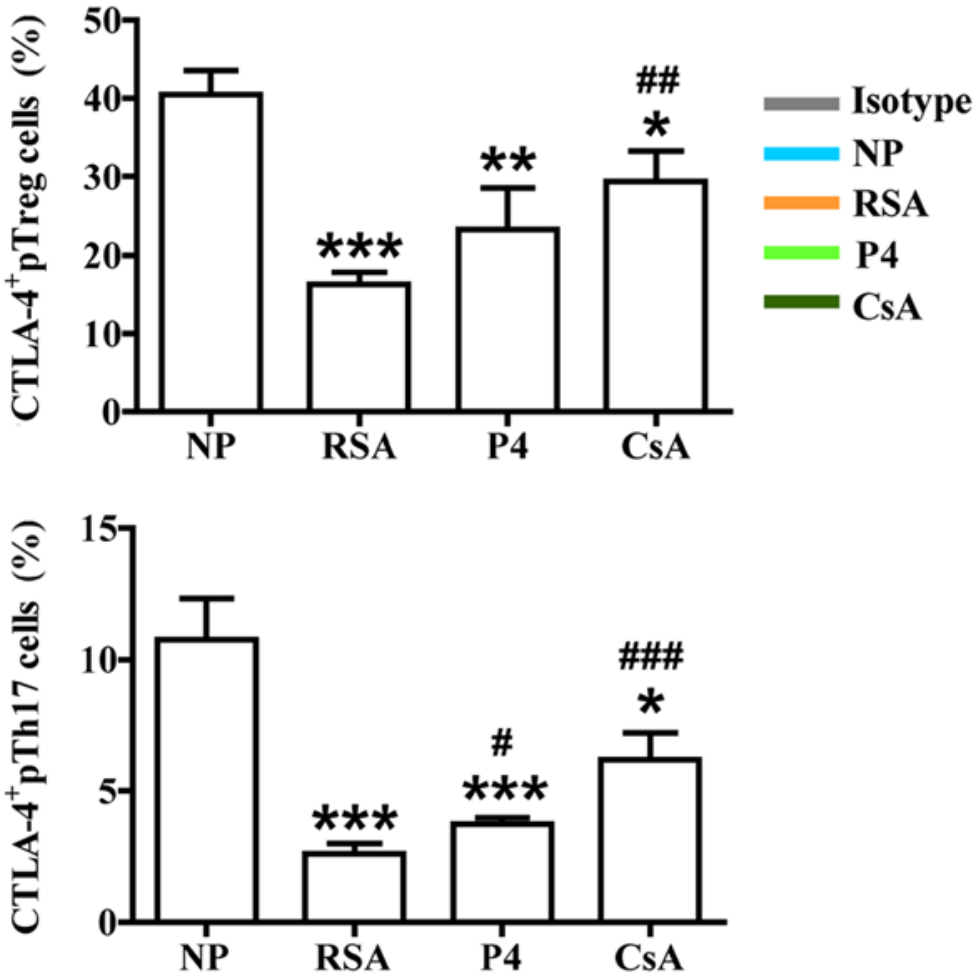

Figure 4. Effects of P4 and CsA on CTLA-4 expression in peripheral blood cells of pregnant females with a history of RSA. Compared with that in the NP group, the production of CTLA-4 in (A) CD4 ${ }^{+}$T cells, (B) Treg cells and (C) Th17 cells in the peripheral blood of subjects in the RSA group was decreased. CsA promoted the expression of CTLA-4 in all three indicated subsets of T cells. P4 also promoted CTLA-4 expression in total CD4+T and Th17 cells. Groups: NP, normal early pregnancy; RSA; patients with RSA; P4, pregnant females with a history of RSA treated with P4; CsA, pregnant females with a history of RSA treated with CsA. ${ }^{*} \mathrm{P}<0.05,{ }^{* *} \mathrm{P}<0.01,{ }^{* * *} \mathrm{P}<0.001$ vs. NP; ${ }^{\#} \mathrm{P}<0.05,{ }^{\# \#} \mathrm{P}<0.01,{ }^{\# \# \#} \mathrm{P}<0.001$ vs. RSA. CsA, cyclosporine A; pTreg cells, peripheral T-regulatory cells; Th17 cells, type-17 T-helper cells; RSA, recurrent spontaneous abortion; P4, progesterone; CTLA, cytotoxic T-lymphocyte-associated protein 4.

function. It has also been indicated that not all Th17 cells are pro-inflammatory, given that Th17 subpopulations with diverse functions may exist (37). IL-10 has been previously associated with non-pathogenic Th17 cells (38), while TGF- $\beta 1$ may be produced mainly by Th17 cells (39). Although P4 had no effect on IL-10 expression in all three indicated subsets of $\mathrm{T}$ cells and TGF- $\beta 1$ expression in $\mathrm{pCD} 4^{+} \mathrm{T}$ cells and pTreg cells, it significantly increased the expression of TGF- $\beta 1$ and CTLA- 4 in pCD $4^{+}$T cells and pTh17 cells, as well as on Tim-3 expression in all three indicated subsets of T cells and PD-1 expression in pTreg cells. As co-stimulatory signals have important roles in T-cell function and pregnancy maintenance $(18,28)$, despite the lack of evidence regarding improved outcomes following first-trimester progesterone therapy in females with a history of RSA $(40,41)$, the results of the present study suggested that P4 may also have an immunomodulatory role through certain co-inhibitory molecules (including Tim-3, PD-1 and CTLA-4) in the treatment of RSA.

The observed association between immunologic abnormalities and RSA has led to the development and use of various types of immune therapy with the objective of restoring normal immune homeostasis during pregnancy. CsA is a previously known immunosuppressive drug that has been used for the treatment of RSA based on previous studies (27,42-48). Previous studies report that CsA: i) Induced $\mathrm{Th} 2$ dominance and Treg-cell proliferation during the implantation window and significantly reduced the embryo absorption rate $(27,42)$; ii) downregulated CD28 expression at the maternal-fetal interface and upregulated CTLA-4 expression in abortion-prone mice (43); 
A

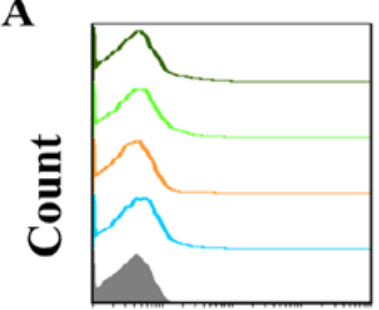

Tim-3

B

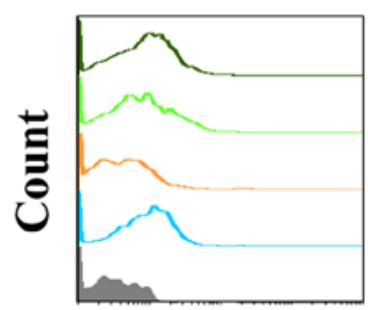

Tim-3

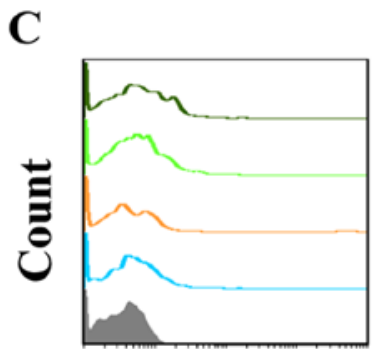

Tim-3
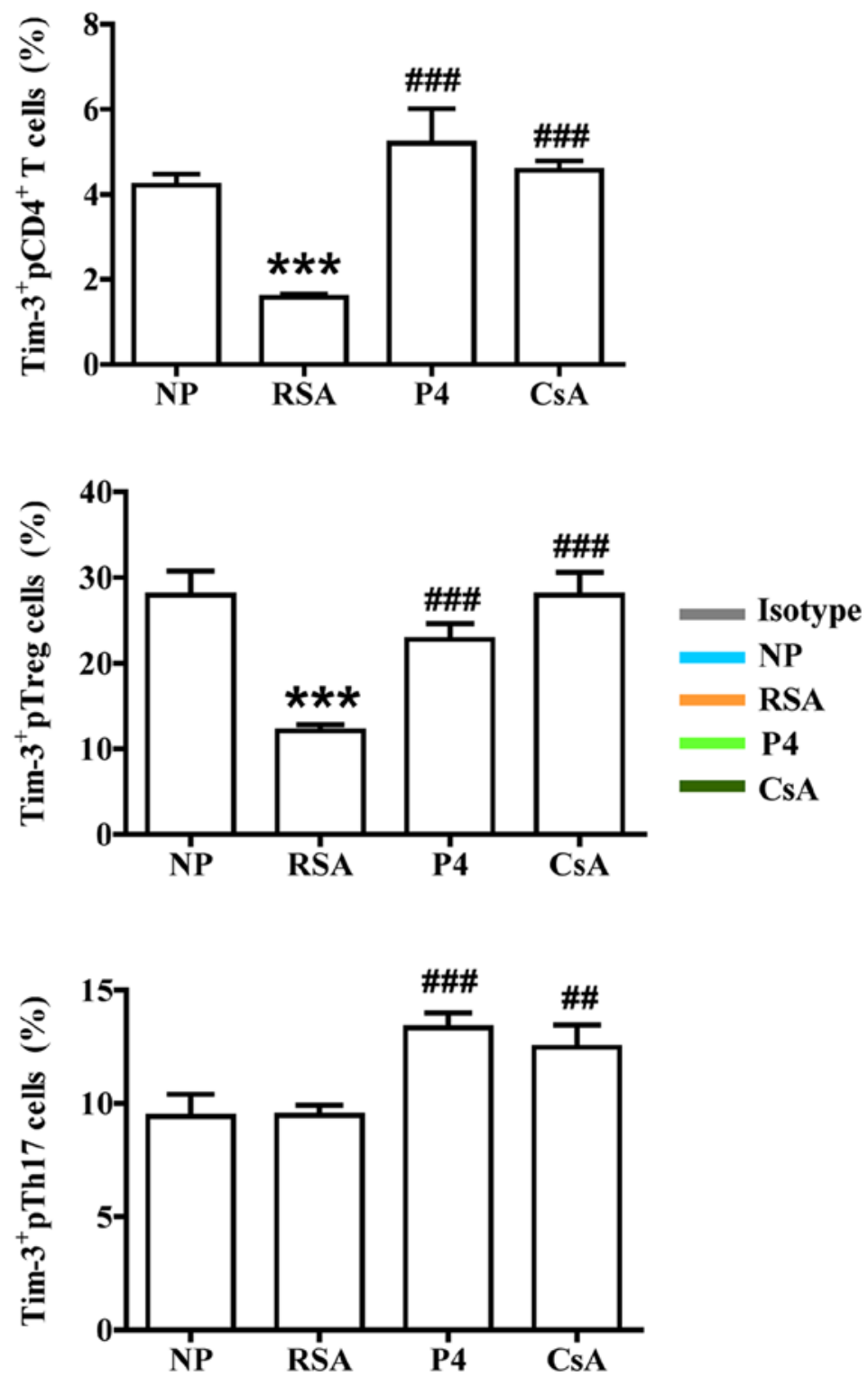

Figure 5. Effects of $\mathrm{P} 4$ and CsA on Tim-3 expression in peripheral blood cells of pregnant females with a history of RSA. Compared with that in the NP group, the production of Tim-3 in (A) CD4 ${ }^{+} \mathrm{T}$ cells and (B) Treg cells in the peripheral blood of subjects in the RSA group was decreased. (C) In Th17 cells in the peripheral blood, no significant difference in Tim-3 expression between the RSA and NP group was determined. P4 and CsA promoted Tim-3 expression in all three indicated subsets of T cells. Groups: NP, normal early pregnancy; RSA; patients with RSA; P4, pregnant females with a history of RSA treated with P4; CsA, pregnant females with a history of RSA treated with CsA. ${ }^{* * * * *} \mathrm{P}<0.001$ vs. NP; ${ }^{\# \#} \mathrm{P}<0.01,{ }^{\# \# \# P} \mathrm{P}<0.001$ vs. RSA. CsA, cyclosporine A; pTreg cells, peripheral T-regulatory cells; Th17 cells, type-17 T-helper cells; RSA, recurrent spontaneous abortion; P4, progesterone; TIM-3, T-cell immunoglobulin mucin family member-3.

iii) improved adhesion and invasion of mouse preimplantation embryos (44); and iv) promoted trophoblastic invasion and proliferation at low dosages $(0.0001-1 \mu \mathrm{mol} / 1)(45,46)$. Small-scale clinical observations $(47,48)$, in addition to unpublished data by our group, have indicated that CsA significantly increased the live birth rate in cases of RSA without increasing complications during pregnancy and among neonates.

The present study suggested that CsA reduced the proportion of Th17 cells and promoted Treg-cell dominance among pregnant females with a history of RSA. CsA decreased the production of IL-17A (the major effector cytokine of Th17 cells) and also upregulated the expression of TGF- $\beta 1$, IL-10, CTLA-4 and Tim-3. These results suggest that $\mathrm{CsA}$, as a trophoblast function promoter, also restores the Th17/Treg-cell balance, while inducing Th2 bias during pregnancy. Accordingly, future research group will be aimed at studying the effect of $\mathrm{P} 4$ and CsA on other pro-inflammatory cytokines in Th17 cells, including IL-6, IL-21 and IL-23. Further investigations are required to explore whether non-pathogenic Th17 subsets and pro-inflammatory Th17 subpopulations co-exist at the maternal-fetal interface, and which mechanisms may be used to modulate Th17 subsets during pregnancy.

To date, RSA with its underlying causes and mechanisms has remained to be fully elucidated. Studies have indicated that the more abortions occur, the higher the likelihood of abortion during the next pregnancy. For instance, after two spontaneous abortions, the risk of spontaneous abortion during the third pregnancy is $\sim 30 \%$, whereas after $>3$ 

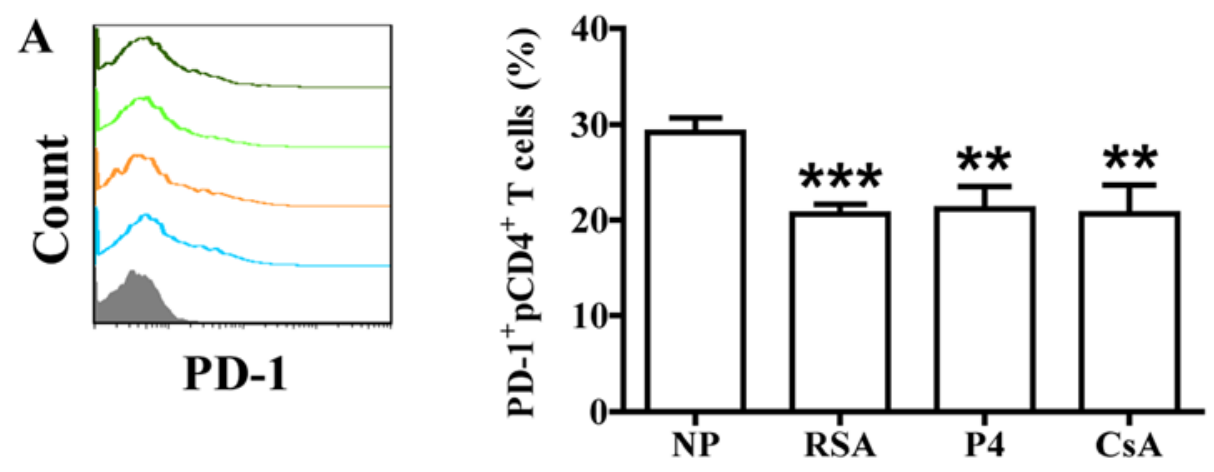

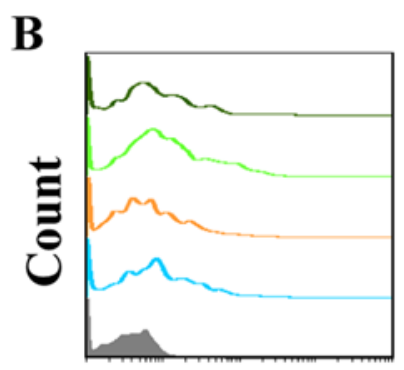

PD-1

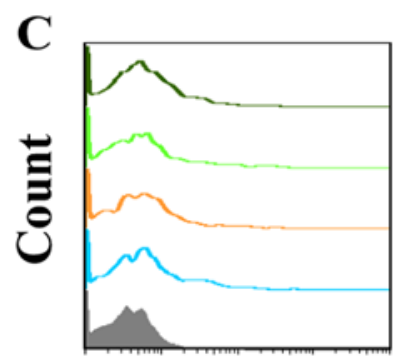

PD-1
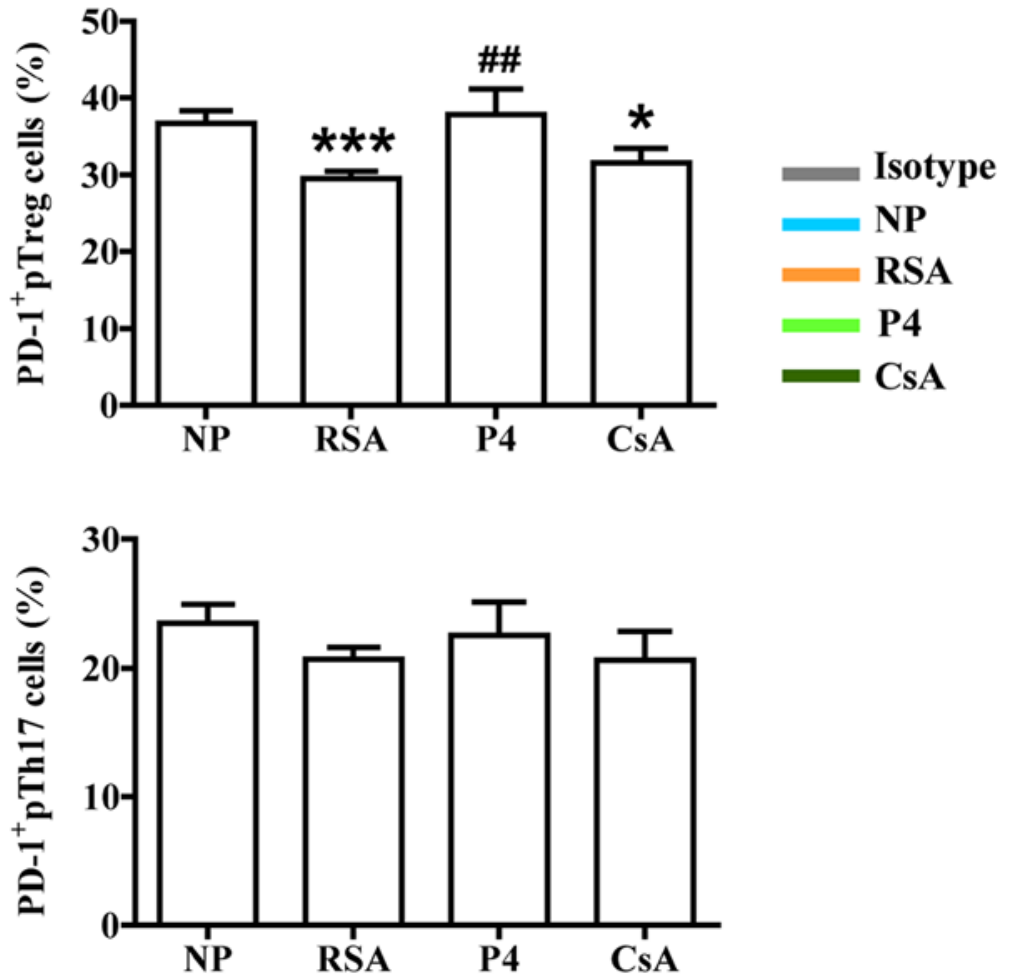

Figure 6. Effects of P4 and CsA on PD-1 expression in peripheral blood cells of pregnant females with a history of RSA. Compared with that in the NP group, the production of PD-1 in (A) CD4 $4^{+} \mathrm{T}$ cells and (B) Treg cells in the peripheral blood of subjects in the RSA group was decreased. (C) In Th17 cells in the peripheral blood, no significant difference in PD-1 expression between the RSA and NP group was determined. While P4 and CsA had no effects on PD-1 expression in total $\mathrm{CD}^{+} \mathrm{T}$ cells and Th17 cells, P4 promoted PD-1 expression in Treg cells. Groups: NP, normal early pregnancy; RSA; patients with RSA; P4, pregnant females with a history of RSA treated with $\mathrm{P} 4$; CsA, pregnant females with a history of RSA treated with CsA. ${ }^{*} \mathrm{P}<0.05,{ }^{* * *} \mathrm{P}<0.01,{ }^{* * * *} \mathrm{P}<0.001$ vs. NP ; ${ }^{\# \#} \mathrm{P}<0.01$ vs. RSA. CsA, cyclosporine A; pTreg cells, peripheral T-regulatory cells; Th17 cells, type-17 T-helper cells; RSA, recurrent spontaneous abortion; P4, progesterone; PD-1, programmed cell death-1.

spontaneous abortions, the risk of spontaneous abortion during the next pregnancy maybe as high as $45 \%$ (49). Early diagnosis and treatment of RSA has been of great significance to families. Although previous studies have focused on the maternal-fetal interface, non-invasive examination is of great importance in clinical practice. The present study investigated the imbalance between Th17 and Treg cells in the peripheral blood to improve the current understanding of the pathogenesis of RSA. The results confirmed that the percentage of Treg cells and the expression of TGF- $\beta 1$, IL-10, CTLA-4 and Tim-3 decreased in the peripheral blood of RSA patients, which may provide an experimental basis for non-invasive monitoring indicators of pregnancy. However, while the experiments were performed using flow cytometry with PMA stimulation, determining the serum levels of cytokines in peripheral blood or cytokine production in different T-cell subpopulations through ELISA or PCR may have been more representative of the in vivo situation. Of course, further follow-up and larger-scale studies are required to confirm whether such molecules maybe used as monitoring indicators of pregnancy. The present results revealed that, while CsA reduced the proportion of Th17 cells and promoted Treg-cell dominance, P4 may also have an immunomodulatory role through certain co-inhibitory molecules to exert its treatment effect in RSA. However, the small sample size and lack of follow-up of pregnancy outcomes have limited the assessment of the effects of P4 and CSA on fetal protection in the present study. However, follow up of the subjects of the present study regarding pregnancy outcomes will continue and, in the future, the sample size will be expanded. 


\section{Acknowledgements}

Not applicable.

\section{Funding}

The present study was supported by grant from the Nature Science Foundation from the National Nature Science Foundation of China (grant nos. 31700799 to SCW, 81630036, 91542116 and 31570920 to MRD, and 81490744 to DJL), the National Basic Research Program of China (grant no. 2015CB943300 to DJL and MRD), the National Key R\&D Program of China (grant no. 2017YFC1001403 to DJL and MRD), the Program of Shanghai Academic/Technology Research Leader (grant no. 17XD1400900 to MRD), the Innovation-oriented Science and Technology Grant from NPFPC Key Laboratory of Reproduction Regulation (grant no. CX2017-2 to MRD), the Shanghai Sailing Program (grant no. 17YF1411600 to SCW), the Training Program for Young Talents of Shanghai Health System (grant no. 2018YQ07 to SCW), the Development Fund of Shanghai Talents (grant no. 2018110 to SCW) and the Shanghai Chenguang Program (grant no. 18CG09 to SCW).

\section{Availability of data and materials}

The datasets used and/or analyzed during the present study are available from the corresponding author on reasonable request.

\section{Authors' contributions}

SCW and MDL carried out the experiments. SCW, DJL and MRD conceived experiments and analyzed data. MDL, CQC, FRS, JFY and JFQ coordinated the sample collection, data interpretation, literature search, and figure preparation. JFQ and SCW drafted the manuscript. MRD revised the manuscript. All authors read and approved the final manuscript.

\section{Ethics approval and consent to participate}

Approval of the present study was obtained from the ethics committee of the Obstetrics and Gynecology Hospital of Fudan University (Shanghai, China) and all subjects provided written informed consent.

\section{Patient consent for publication}

All subjects provided written informed consent for the publication of associated data.

\section{Competing interests}

The authors declare that they have no competing interests.

\section{References}

1. Rai R and Regan L: Recurrent miscarriage. Lancet 368: 601-611, 2006.

2. Arck PC and Hecher K: Fetomaternal immune cross-talk and its consequences for maternal and offspring's health. Nat Med 19: $548-556,2013$
3. Zeng W, Liu Z, Liu X, Zhang S, Khanniche A, Zheng Y, Ma X, Yu T, Tian F, Liu XR, et al: Distinct transcriptional and alternative splicing signatures of decidual $\mathrm{CD}^{+} \mathrm{T}$ cells in early human pregnancy. Front Immunol 8: 682, 2017.

4. Lash GE, Otun HA, Innes BA, Kirkley M, De Oliveira L, Searle RF, Robson SC and Bulmer JN: Interferon-gamma inhibits extravillous trophoblast cell invasion by a mechanism that involves both changes in apoptosis and protease levels. FASEB J 20: 2512-2518, 2006.

5. Chaouat G: The Th1/Th2 paradigm: Still important in pregnancy? Semin Immunopathol 29: 95-113, 2007.

6. Wegmann TG, Lin H, Guilbert L and Mosmann TR: Bidirectional cytokine interactions in the maternal-fetal relationship: Is successful pregnancy a TH2 phenomenon? Immunol Today 7: 353-356, 1993.

7. Crome SQ, Wang AY and Levings MK: Translational mini-review series on Th17 Cells: Function and regulation of human T helper 17 cells in health and disease. Clin Exp Immunol 159: 109-119, 2010.

8. Jutel M, Akdis M, Budak F, Aebischer-Casaulta C, Wrzyszcz M, Blaser K and Akdis CA: IL-10 and TGF-beta cooperate in the regulatory $\mathrm{T}$ cell response to mucosal allergens in normal immunity and specific immunotherapy. Eur J Immunol 33: 1205-1214, 2003.

9. Sheu A, Chan Y, Ferguson A, Bakhtyari MB, Hawke W, White C, Chan YF, Bertolino PJ, Woon HG, Palendira U, et al: A proinflammatory $\mathrm{CD}^{+} \mathrm{T}$ cell phenotype in gestational diabetes mellitus. Diabetologia 61: 1633-1643, 2018.

10. Muyayalo KP, Li ZH, Mor G and Liao AH: Modulatory effect of intravenous immunoglobulin on Th17/Treg cell balance in women with unexplained recurrent spontaneous abortion. Am J ReprodImmunol 80: e13018, 2018.

11. Hosseini A, Dolati S, Hashemi V, Abdollahpour-Alitappeh M and Yousefi M: Regulatory T and T helper 17 cells: Their roles in preeclampsia. J Cell Physiol 233: 6561-6573, 2018.

12. Game DS and Lechler RI: Pathways of allorecognition: Implications for transplantation tolerance. Transpl Immunol 10: 101-108, 2002.

13. Khoury SJ and Sayegh MH: The roles of the new negative $\mathrm{T}$ cell costimulatory pathways in regulating autoimmunity. Immunity 20: 529-538, 2004.

14. Cooksley H, Riva A, Katzarov K, Hadzhiolova-Lebeau T, Pavlova S, Simonova M, Williams R and Chokshi S: Differential expression of immune inhibitory checkpoint signatures on antiviral and inflammatory $\mathrm{T}$ cell populations in chronic hepatitis B. J Interferon Cytokine Res 38: 273-282, 2018.

15. Zhao J, Lin B, Deng H, Zhi X, Li Y, Liu Y, Bible PW, Li Q, $\mathrm{Xu}$ B, Wei L, et al: Decreased expression of TIM-3 on Th17 cells associated with ophthalmopathy in patients with Graves' disease. Curr Mol Med 18: 83-90, 2018.

16. Wei CB, Tao K, Jiang R, Zhou LD, Zhang QH and Yuan CS: Quercetin protects mouse liver against triptolide-induced hepatic injury by restoring Th17/Treg balance through Tim-3 and TLR4-MyD88-NF- $\mathrm{BB}$ pathway. Int Immunopharmacol 53: 73-82, 2017.

17. Zhang Y, Liu Z, Tian M, Hu X, Wang L, Ji J and Liao A: The altered PD-1/PD-L1 pathway delivers the 'one-two punch' effects to promote the Treg/Th17 imbalance in pre-eclampsia. Cell Mol Immunol 15: 710-723, 2018.

18. D'Addio F, Riella LV, Mfarrej BG, Chabtini L, Adams LT, Yeung M, Yagita H, Azuma M, Sayegh MH and Guleria I: The link between the PDL1 costimulatory pathway and Th17 in fetomaternal tolerance. J Immunol 187: 4530-4541, 2011.

19. Liao H, Peng X, Gan L, Feng J, Gao Y, Yang S, Hu X, Zhang L, Yin $\mathrm{Y}$, Wang $\mathrm{H}$ and $\mathrm{Xu} \mathrm{X}$ : Protective regulatory $\mathrm{T}$ cell immune response induced by intranasal immunization with the live-attenuated pneumococcal vaccine SPY1 via the transforming growth factor- $\beta 1-S m a d 2 / 3$ pathway. Front Immunol 9: 1754, 2018.

20. Kawai K, Uchiyama M, Hester J, Wood K and Issa F: Regulatory T cells for tolerance. Hum Immunol 79: 294-303, 2018.

21. Gupta S, Thornley TB, Gao W, Larocca R, Turka LA, Kuchroo VK and Strom TB: Allograft rejection is restrained by short-lived TIM-3+PD-1+Foxp3+ tregs. J Clin Invest 122: 2395-2404, 2012.

22. Wang S, Zhu X, Xu Y, Zhang D, Li Y, Tao Y, Piao H, Li D and Du M: Programmed cell death-1 (PD-1) and T-cell immunoglobulin mucin-3 (Tim-3) regulate CD4+ T cells to induce type 2 helper T Cell (Th2) bias at the maternal-fetal interface. Hum Reprod 31: 700-711, 2016.

23. Wang S, Chen C, Li M, Qian J, Sun F, Li Y, Yu M, Wang M, Zang X, Zhu R, et al: Blockade of CTLA-4 and Tim-3 pathways induces fetal loss with altered cytokine profiles by decidual CD4 ${ }^{+} \mathrm{T}$ cells. Cell Death Dis 10: 15, 2019. 
24. Spencer TE and Bazer FW: Biology of progesterone action during pregnancy recognition and maintenance of pregnancy. Front Biosci 7: d1879-d1898, 2002.

25. Arck P, Hansen PJ, Mulac Jericevic B, Piccinni MP and Szekeres-Bartho J: Progesterone during pregnancy: Endocrineimmune cross talk in mammalian species and the role of stress. Am J Reprod Immunol 58: 268-279, 2007.

26. Sketris I, Yatscoff R, Keown P, Canafax DM, First MR, Holt DW, Schroeder TJ and Wright M: Optimizing the use of cyclosporine in renal transplantation. Clin Biochem 28: 195-211, 1995.

27. Du MR, Dong L, Zhou WH, Yan FT and Li DJ: Cyclosporin a improves pregnancy outcome by promoting functions of trophoblasts and inducing maternal tolerance to the allogeneic fetus in abortion-prone matings in the mouse. Biol Reprod 76: 906-914, 2007.

28. Xu YY, Wang SC, Li DJ and Du MR: Co-signaling molecules in maternal-fetal immunity. Trends Mol Med 23: 46-58, 2017.

29. Di Renzo GC, Giardina I, Clerici G, Brillo E and Gerli S: Progesterone in Normal and Pathological Pregnancy. Horm Mol Biol Clin Investig 27: 35-48, 2016.

30. Chien EJ, Chang CP, Lee WF, Su TH and Wu CH: Non-genomic immunosuppressive actions of progesterone inhibits PHA-induced alkalinization and activation in T cells. J Cell Biochem 99: 292-304, 2006.

31. Piccinni MP, Giudizi MG, Biagiotti R, Beloni L, Giannarini L, Sampognaro S, Parronchi P, Manetti R, Annunziato F, Livi C, et al: Progesterone favors the development of human $\mathrm{T}$ helper cells producing Th2-type cytokines and promotes both IL-4 production and membrane CD30 expression in established Th1 cell clones. J Immunol 155: 128-133, 1995.

32. Mjosberg J, Svensson J, Johansson E, Hellström L, Casas R, Jenmalm MC, Boij R, Matthiesen L, Jönsson JI, Berg G and Ernerudh J: Systemic reduction of functionally suppressive CD4dimCD25highFoxp3+ Tregs in human second trimester pregnancy is induced by progesterone and 17beta-estradiol J Immunol 183: 759-769, 2009.

33. Lee JH, Ulrich B, Cho J, Park J and Kim CH: Progesterone promotes differentiation of human cord blood fetal T cells into T regulatory cells but suppresses their differentiation into Th17 cells. J Immunol 187: 1778-1787, 2011.

34. Fu B, Tian Z and Wei H: TH17 Cells in human recurrent pregnancy loss and pre-eclampsia. Cell Mol Immunol 11: 564-570, 2014.

35. Wang S, Qian J, Sun F, Li M, Ye J, Li M, Du M and Li D Bidirectional regulation between 1st trimester HTR8/SVneo trophoblast cells and in vitro differentiated Th17/Treg cells suggest a fetal-maternal regulatory loop in human pregnancy. Am J Reprod Immunol 81: e13106, 2019.

36. Wu HX, Jin LP, Xu B, Liang SS and Li DJ: Decidual stromal cells recruit Th17 cells into decidua to promote proliferation and invasion of human trophoblast cells by secreting IL-17. Cell Mol Immunol 11: 253-262, 2014.
37. Lee Y, Awasthi A, Yosef N, Quintana FJ, Xiao S, Peters A, Wu C, Kleinewietfeld M, Kunder S, Hafler DA, et al: Induction and molecular signature of pathogenic TH17 cells. Nat Immunol 13: 991-999, 2012

38. McGeachy MJ, Bak-Jensen KS, Chen Y, Tato CM,Blumenschein W, McClanahan T and Cua D: TGF-beta and IL-6 drive the production of IL-17 and IL-10 by T cells and restrain T(H)-17 cell-mediated pathology. Nat Immunol 8: 1390-1397, 2007.

39. Gutcher I,Donkor MK,MaQ, Rudensky AY,Flavell RA and LiMO Autocrine transforming growth factor- $\beta 1$ promotes in vivo Th17 cell differentiation. Immunity 34: 396-408, 2011.

40. Coomarasamy A, Williams H, Truchanowicz E, Seed PT, Small R, Quenby S, Gupta P, Dawood F, Koot YE, Bender Atik R, et al: A randomized trial of progesterone in women with recurrent miscarriages. N Engl J Med 373: 2141-2148, 2015.

41. Coomarasamy A, Williams H, Truchanowicz E, Seed PT, Small R, Quenby S, Gupta P, Dawood F, Koot YE, Atik RB, et al: PROMISE: First-trimester progesterone therapy in women with a history of unexplained recurrent miscarriages-a randomised, double-blind, placebo-controlled, international multicentre trial and economic evaluation. Health Technol Assess 20: 1-92, 2016.

42. Piao HL, Wang SC, Tao Y, Zhu R, Sun C, Fu Q, Du MR and Li DJ: Cyclosporine A enhances Th2 bias at the maternal-fetal interface in early human pregnancy with aid of the interaction between maternal and fetal cells. PLoS One 7: e45275, 2012.

43. Zhou WH, Dong L, Du MR, Zhu XY and Li DJ: Cyclosporin a improves murine pregnancy outcome in abortion-prone matings: Involvement of CD80/86 and CD28/CTLA-4. Reproduction 135 : 385-395, 2008

44. Huang Y, Ma Y, Ma L, Mao JL, Zhang Y, Du MR and Li DJ: Cyclosporine A improves adhesion and invasion of mouse preimplantation embryos via upregulating integrin $\beta 3$ and matrix metalloproteinase-9. Int J Clin Exp Patho 7: 1379-1388, 2014

45. Du MR, Zhou WH, Yan FT, Zhu XY, He YY, Yang JY and Li DJ: Cyclosporine A induces titin expression via MAPK/ERK signalling and improves proliferative and invasive potential of human trophoblast cells. Hum Reprod 22: 2528-2537, 2007.

46. Zhou WH, Du MR, Dong L, Zhu XY, Yang JY, He YY and Li DJ: Cyclosporina increases expression of matrix metalloproteinase 9 and 2 and invasiveness in vitro of the first-trimester human trophoblast cells via the mitogen-activated protein kinase pathway. Hum Reprod 22: 2743-2750, 2007.

47. Fu JH: Analysis of the use of cyclosporina to treat refractory immune recurrent spontaneous abortion. Clin Exp Obstet Gynecol 42: 739-742, 2015

48. Ling Y, Huang Y, Chen C, Mao J and Zhang H: Low dose cyclosporin a treatment increases live birth rate of unexplained recurrent abortion-initial cohort study. Clin Exp Obstet Gynecol 44: 230-235, 2017.

49. Martínez-Zamora MÁ, Cervera R and Balasch J: Recurrent miscarriage, antiphospholipid antibodies and the risk of thromboembolic disease. Clin Rev Allergy Immunol 43: 265-274, 2012. 